\title{
Hall Effect on ECF Flow along a Rotating Infinite Porous Plate in the Presence of Transverse Magnetic Field
}

\author{
Avijit Podder, Md. Mahmud Alam* \\ Mathematics Discipline, Science, Engineering and Technology School, Khulna University, Khulna, Bangladesh \\ Email: *alam_mahmud2000@yahoo.com
}

How to cite this paper: Podder, A. and Alam, Md.M. (2021) Hall Effect on ECF Flow along a Rotating Infinite Porous Plate in the Presence of Transverse Magnetic Field. Open Journal of Applied Sciences, 11, 312-326.

https://doi.org/10.4236/ojapps.2021.113023

Received: January 20, 2021

Accepted: March 20, 2021

Published: March 23, 2021

Copyright $\odot 2021$ by author(s) and Scientific Research Publishing Inc. This work is licensed under the Creative Commons Attribution International License (CC BY 4.0).

http://creativecommons.org/licenses/by/4.0/

\begin{abstract}
Unsteady extracellular fluid (ECF) flow along with a rotating infinite vertical porous plate in the presence of a transverse magnetic field has been studied numerically. The dimensional governing equations have been non-dimensionalized by useful dimensionless variables. The explicit finite difference method has been used to solve dimensionless equations. The numerical results have been calculated by studio developer FORTRAN 6.6a and MATLAB 2018a. For perfect conducting case, Magnetic Diffusivity Parameter $5 \leq P_{m} \leq 15$ has been taken in induction equation. For good accuracy, stability and convergence analysis have been analyzed. Mesh Sensitivity test, steady-state solution test, and code validation test have been performed. For time step $\tau=1$, the numerical results have been found for the primary velocity, secondary velocity, angular velocity, primary induced magnetic field, secondary induced magnetic field, temperature as well as shear stresses along $x$ and $z$ direction, couple stress along $z$ direction, current densities along $x$ and $z$ direction and the Nusselt number. Finally, the effects of various parameters have been separately discussed and illustrated graphically.
\end{abstract}

\section{Keywords}

Extracellular Fluid, MHD, Heat Transfer, Explicit Finite Difference

Technique, Transverse Magnetic Field

\section{Introduction}

Extracellular fluids are perfectly conducting fluids of microstructure that exhibit the micro-rotational effects and micro-rotational inertia. The enthusiasm of the cell by any physical effect causes a nerve impulse, sodium ions are pumped in 
the cell, potassium ions are pumped out, and the cell membrane reaches a depolarization state at which the electric signals are transmitted from one cell to another when the action potential is conducted at speeds that range from 1 to $100 \mathrm{~m} \cdot \mathrm{s}^{-1}$, indicates the impulse moves along the fiber, which was found by Hodgkin et al. [1]. The Nobel Prize for physiology or medicine was awarded in 1963 for formulating these ionic mechanisms involved in nerve cell activity. Since the liquid sodium $\mathrm{Na}_{(l)}\left(100^{\circ} \mathrm{C}\right)$ and liquid potassium $\mathrm{K}_{(l)}\left(100^{\circ} \mathrm{C}\right)$ exhibit very small electrical resistivity $\left(\rho_{L}(\exp )=9.6 \times 10^{-6} \Omega \cdot \mathrm{cm}\right)$ and $\left(\rho_{L}(\exp )=12.97 \times 10^{-6} \Omega \cdot \mathrm{cm}\right)$, respectively, the extracellular fluid can be considered as a perfect conducting micropolar fluid [2].

Eringen [3] first formulated the theory of micropolar fluids, which display the effect of local rotary inertia and couple stress. Connection with the blood rheology interesting results has been reported by Kline and Alien [4]. Low concentration suspension flow for micropolar fluid has been pointed out by many investigators as noticed in Ariman [5]. Ariman et al. [6] also investigated the microcontinuum fluid mechanics. Eringen [7] extended the micropolar fluid theory and developed the theory of thermo-micro fluids. The effect of Hall currents on free convective flow through a porous medium bounded by an infinite vertical plate has been studied by Ram [8]. Combined effects of thermal radiations and Hall current on moving vertical porous plate in a rotating system with variable temperature have been analyzed by Garg [9]. The boundary layer flows in the presence of Soret and Dufour effects associated with the thermal-diffusion and diffusion-thermo for the mix convection have been analyzed by Kafoussias and Williams [10]. The influence of magnetic field on heat and mass transfer by natural convection from vertical surfaces in porous media considering Soret and Dufour effects have been studied by Postelnicu [11]. Mohammadein et al. [12] formulated a model to show the effects of transverse magnetic field in a micropolar fluid. A boundary layer theory for micropolar has been established by Peddiesen et al. [13]. The effect of Hall current, Soret, and Dufour effects on MHD flow by mixed convection over a vertical surface in porous media has been studied by Shateyi et al. [14]. MHD flow over a moving plate in a rotating fluid with magnetic field and Hall current has been investigated by Takhar et al. [15]. Free convection effects on perfectly conducting fluid have been formulated by Ezzat [16]. Numerical simulation and stability analysis on MHD free convective heat and mass transfer unsteady flow through a porous medium in a rotating system with the induced magnetic field have been investigated by Haque et al. [17]. Finite difference solution of MHD mixed convection flow with heat generation and the chemical reaction has been investigated by Ahmed et al. [18].

In the present work, our aim is to simulate the Hall current effect on ECF flow along with a rotating infinite vertical porous plate in the presence of the transverse magnetic field. The basic governing equations of the problem have been non-dimensionalized by using dimensionless variables. The obtained dimensionless equations have been solved numerically using the explicit finite difference method (FDM). Stability and convergence analysis has been analyzed to 
obtain a converged solution. Finally, all possible outcomes have been illustrated.

\section{Mathematics Formulation}

An unsteady extracellular fluid flow along with an infinite vertical porous plate with transverse magnetic field, joule heating, viscous dissipation effect in a rotating system are considered. The positive $x$ coordinate is measured along with the plate in the direction of fluid motion and the $y$ coordinate is measured normal to the plate. Initially, it is considered that the plate, as well as the fluid, are at the same temperature $T\left(=T_{\infty}\right)$. Also, it is assumed that the fluid and the plate are at rest after that the plate is to be moving with a constant velocity $U_{\infty}$. Instantaneously at time $t>0$, the temperature of the plate raised to $T_{w}\left(>T_{\infty}\right)$, where $T_{w}$ is the temperature of the wall and $T_{\infty}$ is the temperature outside the plate and the system is rotating anticlockwise with rotational velocity $\Omega$. The physical problem is furnished in Figure 1.

A strong uniform magnetic field $H_{0}$ can be taken as $\left(0, H_{0}, 0\right)$. For extracellular fluid, magnetic Reynolds number $R_{m} \gg 1$ which implies dimensionless Magnetic Diffusivity Number $\left(P_{m}\right)$ values between 5 to 15 . The divergence equation $\nabla \cdot \boldsymbol{H}=0$ of Maxwell's equation for the magnetic field gives $H_{y}=H_{0}$. Using the relation $\nabla \cdot \boldsymbol{J}=0$ for the current density $\boldsymbol{J}=\left(J_{x}, J_{y}, J_{z}\right)$ implies $J_{y}=$ constant . Since the plate is non-conducting, $J_{y}=0$ at the plate and hence zero everywhere. The fluid velocity vector is given by $\boldsymbol{q}=(u, v, w)$.

The non-dimensional variables that have been used in the governing equations are as follows,

$$
\begin{gathered}
\tau=\frac{t U_{\infty}^{2}}{v}, Y=\frac{y U_{\infty}}{v}, \quad U=\frac{u}{U_{\infty}}, \quad V=\frac{v}{U_{\infty}}, \quad W=\frac{w}{U_{\infty}}, \quad N=\frac{v N_{z}}{U_{\infty}^{2}}, \\
H_{1}=\sqrt{\frac{\mu}{\rho}} \frac{H_{x}}{U_{\infty}}, \quad H_{3}=\sqrt{\frac{\mu}{\rho}} \frac{H_{z}}{U_{\infty}} \text { and } \theta=\frac{T-T_{\infty}}{T_{w}-T_{\infty}} .
\end{gathered}
$$

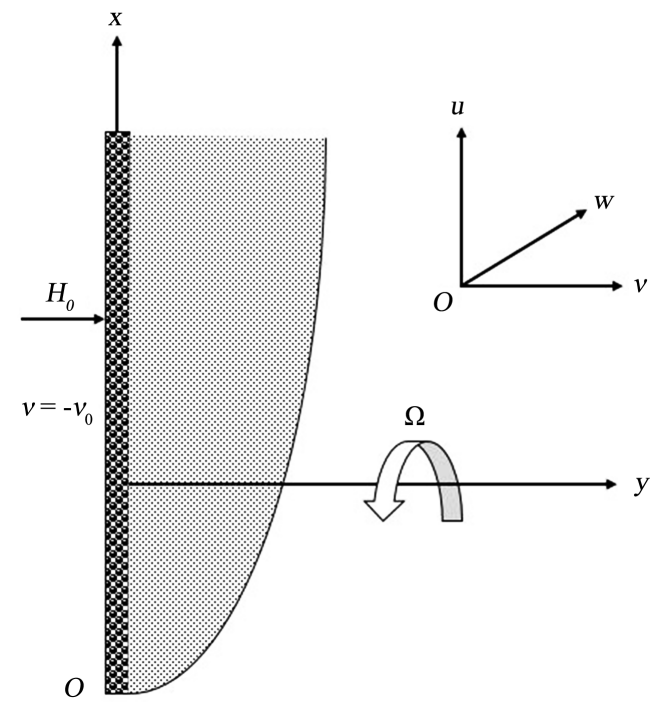

Figure 1. Physical configuration and coordinate system. 
Using the above non-dimensional variables that have been used in the governing equations are as follows,

$$
\begin{gathered}
\frac{\partial V}{\partial Y}=0 \\
\frac{\partial U}{\partial \tau}-S_{u} \frac{\partial U}{\partial Y}=G_{r} \theta+(1+\Delta) \frac{\partial^{2} U}{\partial Y^{2}}+\Delta \frac{\partial N}{\partial Y}+M \frac{\partial H_{1}}{\partial Y}-\frac{U}{D_{a}}-R W \\
\frac{\partial W}{\partial \tau}-S_{u} \frac{\partial W}{\partial Y}=(1+\Delta) \frac{\partial^{2} W}{\partial Y^{2}}+M \frac{\partial H_{3}}{\partial Y}-\frac{W}{D_{a}}+R U \\
\frac{\partial N}{\partial \tau}-S_{u} \frac{\partial N}{\partial Y}=\Lambda \frac{\partial^{2} N}{\partial Y^{2}}-\lambda \frac{\partial U}{\partial Y}-2 \lambda N \\
\frac{\partial H_{1}}{\partial \tau}-S_{u} \frac{\partial H_{1}}{\partial Y}=\frac{1}{P_{m}} \frac{\partial^{2} H_{1}}{\partial Y^{2}}+M \frac{\partial U}{\partial Y}-\frac{\beta_{e}}{P_{m}} \frac{\partial^{2} H_{3}}{\partial Y^{2}} \\
\frac{\partial H_{3}}{\partial \tau}-S_{u} \frac{\partial H_{3}}{\partial Y}=\frac{1}{P_{m}} \frac{\partial^{2} H_{3}}{\partial Y^{2}}+M \frac{\partial W}{\partial Y}+\frac{\beta_{e}}{P_{m}} \frac{\partial^{2} H_{1}}{\partial Y^{2}} \\
\frac{\partial \theta}{\partial \tau}-S_{u} \frac{\partial \theta}{\partial Y}=\frac{1}{P_{r}} \frac{\partial^{2} \theta}{\partial Y^{2}}+\frac{E_{c}}{P_{m}}\left\{\left(\frac{\partial H_{3}}{\partial Y}\right)^{2}+\left(\frac{\partial H_{1}}{\partial Y}\right)^{2}\right\} \\
+E_{c}\left\{\left(\frac{\partial U}{\partial Y}\right)^{2}+\left(\frac{\partial W}{\partial Y}\right)^{2}\right\}+\beta \theta^{p}
\end{gathered}
$$

The corresponding non-dimensional boundary conditions are as follows,

$$
\begin{gathered}
U=1, V=0, W=0, N=-S \frac{\partial U}{\partial Y}, H_{1}=\sqrt{\frac{\mu_{e}}{\rho}} \frac{H_{w}}{U_{\infty}}=1(\text { say }), H_{3}=0, \theta=1 \text { at } Y=0 \\
U=0, V=0, W=0, N=0, H_{1}=0, H_{3}=0, \theta \rightarrow 0 \text { as } Y \rightarrow \infty
\end{gathered}
$$

where $\tau$ represents the dimensionless time, $Y$ is dimensionless Cartesian coordinates, $U, V$ and $W$ are dimensionless velocity components, $H_{1}$ and $H_{3}$ are dimensionless primary and secondary induced magnetic field, $\theta$ is the dimensionless temperature, $G_{r}=\frac{g B_{T}\left(T_{w}-T_{\infty}\right) v}{U_{\infty}^{3}}$ (Grashof Number), $\Delta=\frac{k}{\mu}$ (Microrotational Number), $S_{u}=\frac{v_{0}}{U_{\infty}}$ (Suction Parameter), $M=\frac{1}{4 \pi} \frac{H_{0}}{U_{\infty}} \sqrt{\frac{\mu_{e}}{\rho}}$ (Magnetic Parameter), $D_{a}=\frac{k^{\prime} U_{\infty}^{2}}{v^{2}}$ (Darcy Number), $R=\frac{2 v \Omega}{U_{\infty}^{2}}$ (Rotational Parameter), $\Lambda=\frac{\gamma}{j \mu}$ (Spin Gradient Viscosity), $\lambda=\frac{k v}{\rho j U_{\infty}^{2}}$ (Vortex Viscosity), $P_{r}=\frac{\rho c_{p} v}{\kappa}$ (Prandtl Number), $E_{c}=\frac{U_{\infty}^{2}}{c_{p}\left(T_{w}-T_{\infty}\right)}$ (Eckert Number), $P_{m}=4 \pi \sigma v \mu_{e}$ (Magnetic Diffusivity Number) and $\beta=\frac{Q v\left(T_{w}-T_{\infty}\right)^{p-1}}{\rho c_{p} U_{\infty}^{2}}$ (Heat Generation or Absorption Parameter). 


\section{Shear Stresses, Couple Stress, Current Densities and Nusselt Number}

The shear stress along $x$ direction, $\tau_{x}=\{\mu+(1-S) K\}\left(\frac{\partial u}{\partial y}\right)_{y=0}$ and the shear stress along $z$ direction, $\tau_{z}=\{\mu+(1-S) K\}\left(\frac{\partial w}{\partial y}\right)_{y=0}$ which are proportional to $\left(\frac{\partial U}{\partial Y}\right)_{Y=0}$ and $\left(\frac{\partial W}{\partial Y}\right)_{Y=0}$ respectively.

Couple stress $=\frac{v j \rho}{\gamma}\left(\frac{\partial N}{\partial y}\right)_{y=0}$ which is proportional to $\left(\frac{\partial N}{\partial Y}\right)_{Y=0}$.

Current densities along $x$ and $z$ directions are $J_{x}=\mu\left(-\frac{\partial H_{x}}{\partial y}\right)_{y=0}$ and $J_{z}=\mu\left(-\frac{\partial H_{z}}{\partial y}\right)_{y=0}$ which are proportional to $\left(-\frac{\partial H_{1}}{\partial Y}\right)_{Y=0}$ and $\left(-\frac{\partial H_{3}}{\partial Y}\right)_{Y=0}$ respectively.

Nusselt Number $=\mu\left(-\frac{\partial T}{\partial y}\right)_{y=0}$ which is proportional to $\left(-\frac{\partial \theta}{\partial Y}\right)_{Y=0}$.

\section{Numerical Technique}

In this section, the governing second-order non-linear dimensionless partial differential equations with initial and boundary conditions have been solved. The explicit finite difference method has been used to solve dimensionless equations subject to the boundary conditions. The region with the boundary layer is divided by some perpendicular line of $Y$-axis, where $Y$-axis is normal to the medium as shown in Figure 2.

Here assumed $Y_{\max }(=40)$ i.e. $Y$ varies from 0 to 40 . There is $n(=725)$ grid spacing in the $Y$ direction as shown in Figure 2. And $\Delta Y$ are constant mesh size along $Y$ direction and taken as follows, $\Delta Y=0.055$ (approx.) with the smaller time-step, $\Delta \tau=0.0005$.

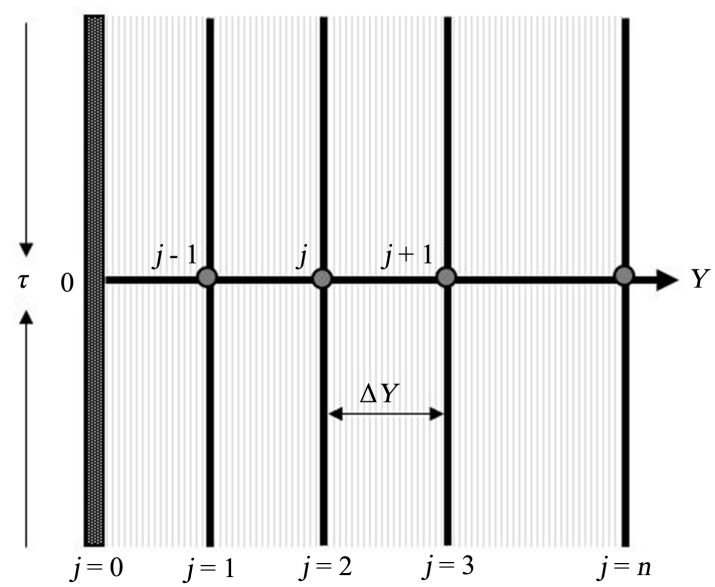

Figure 2. Explicit finite difference system grid. 
Let $U^{\prime}, W^{\prime}, N^{\prime}, H_{1}^{\prime}, H_{3}^{\prime}$ and $\theta^{\prime}$ denote the values of $U, W, N, H_{1}, H_{3}$ and $\theta$ are the end of a time-step respectively. Using the explicit finite difference approximation, the system of partial differential equations (9)-(15) is obtained by approximate set of finite difference equations:

$$
\begin{aligned}
& \frac{V_{i, j+1}-V_{i, j}}{\Delta Y}=0 \\
& \frac{U_{i, j}^{\prime}-U_{i, j}}{\Delta \tau}-S_{u} \frac{U_{i, j+1}-U_{i, j}}{\Delta Y} \\
& =G_{r} \theta_{i, j}+(1+\Delta) \frac{U_{i, j+1}-2 U_{i, j}+U_{i, j-1}}{(\Delta Y)^{2}}+\Delta \frac{N_{i, j+1}-N_{i, j}}{\Delta Y} \\
& +M \frac{H_{1 i, j+1}-H_{1 i, j}}{\Delta Y}-\frac{U_{i, j}}{D_{a}}-R W_{i, j} \\
& \frac{W_{i, j}^{\prime}-W_{i, j}}{\Delta \tau}-S_{u} \frac{W_{i, j+1}-W_{i, j}}{\Delta Y} \\
& =(1+\Delta) \frac{W_{i, j+1}-2 W_{i, j}+W_{i, j-1}}{(\Delta Y)^{2}}+M \frac{H_{3 i, j+1}-H_{3 i, j}}{\Delta Y}-\frac{W_{i, j}}{D_{a}}+R U_{i, j} \\
& \frac{N_{i, j}^{\prime}-N_{i, j}}{\Delta \tau}-S_{u} \frac{N_{i, j+1}-N_{i, j}}{\Delta Y} \\
& =\Lambda \frac{N_{i, j+1}-2 N_{i, j}+N_{i, j-1}}{(\Delta Y)^{2}}-\lambda \frac{U_{i, j+1}-U_{i, j}}{\Delta Y}-2 \lambda N_{i, j} \\
& \frac{H_{1 i, j}^{\prime}-H_{1 i, j}}{\Delta \tau}-S_{u} \frac{H_{1 i, j+1}-H_{1 i, j}}{\Delta Y} \\
& =\frac{1}{P_{m}} \frac{H_{1 i, j+1}-2 H_{1 i, j}+H_{1 i, j-1}}{(\Delta Y)^{2}}+M \frac{U_{i, j+1}-U_{i, j}}{\Delta Y} \\
& -\frac{\beta_{e}}{P_{m}} \frac{H_{3 i, j+1}-2 H_{3 i, j}+H_{3 i, j-1}}{(\Delta Y)^{2}} \\
& \frac{H_{3 i, j}^{\prime}-H_{3 i, j}}{\Delta \tau}-S_{u} \frac{H_{3 i, j+1}-H_{3 i, j}}{\Delta Y} \\
& =\frac{1}{P_{m}} \frac{H_{3 i, j+1}-2 H_{3 i, j}+H_{3 i, j-1}}{(\Delta Y)^{2}}+M \frac{W_{i, j+1}-W_{i, j}}{\Delta Y} \\
& +\frac{\beta_{e}}{P_{m}} \frac{H_{1 i, j+1}-2 H_{1 i, j}+H_{1 i, j-1}}{(\Delta Y)^{2}} \\
& \frac{\theta_{i, j}^{\prime}-\theta_{i, j}}{\Delta \tau}-S_{u} \frac{\theta_{i, j+1}-\theta_{i, j}}{\Delta Y} \\
& =\frac{1}{P_{r}} \frac{\theta_{i, j+1}-2 \theta_{i, j}+\theta_{i, j-1}}{(\Delta Y)^{2}}+\frac{E_{c}}{P_{m}}\left\{\left(\frac{H_{3 i, j+1}-H_{3 i, j}}{\Delta Y}\right)^{2}+\left(\frac{H_{1 i, j+1}-H_{1 i, j}}{\Delta Y}\right)^{2}\right\} \\
& +E_{c}\left\{\left(\frac{U_{i, j+1}-U_{i, j}}{\Delta Y}\right)^{2}+\left(\frac{W_{i, j+1}-W_{i, j}}{\Delta Y}\right)^{2}\right\}+\beta \theta_{i, j}^{p}
\end{aligned}
$$

and the boundary condition becomes: 


$$
\begin{gathered}
U_{0}^{n}=1, V_{0}^{n}=0, W_{0}^{n}=0, N_{0}^{n}=-S \frac{\partial U_{0}^{n}}{\partial Y}, H_{10}^{n}=1(\text { say }), H_{30}^{n}=0, \theta_{0}^{n}=1 \text { at } Y=0 \\
U_{L}^{n}=0, V_{L}^{n}=0, W_{L}^{n}=0, N_{L}^{n}=0, H_{1 L}^{n}=0, H_{3 L}^{n}=0, \theta_{L}^{n} \rightarrow 0 \text { as } Y=L \rightarrow \infty
\end{gathered}
$$

Here $p$ is the power of heat generation parameter and $S$ indicates constant value which represents different cases. When $S=0$, we obtain $N=0$ which represents no-spin condition, i.e., the microelements in a concentrated particle flow close to the wall are not able to rotate. The case $S=\frac{1}{2}$ represents vanishing of the anti-symmetric part of the stress tensor and represents weak concentration. The case $S=1$ represents turbulent boundary layer flow. The subscript $j$ designates the grid points with $Y$ coordinates and the superscript $n$ represents a value of time, $\tau=n \Delta \tau$ where $n=0,1,2, \cdots$. The primary velocity ( $U$ ), secondary velocity $(W)$, angular velocity $(N)$, primary induced magnetic field $\left(H_{1}\right)$, secondary induced magnetic field $\left(\mathrm{H}_{3}\right)$ and temperature $(\theta)$ distributions at all interior nodal points may be computed by successive applications of the above finite difference equations. The numerical values of the shear stresses, couple stress, current densities and Nusselt number are evaluated by Three-Point formula.

The stability conditions of the problem are furnished as follows:

$$
\begin{gathered}
\frac{\Delta \tau}{2 D_{a}}+2(1+\Delta) \frac{\Delta \tau}{(\Delta Y)^{2}}+S_{u} \frac{\Delta \tau}{\Delta Y} \leq 1, \Delta \tau \lambda+S_{u} \frac{\Delta \tau}{\Delta Y}+2 \Lambda \frac{\Delta \tau}{(\Delta Y)^{2}} \leq 1 \\
S_{u} \frac{\Delta \tau}{\Delta Y}+\frac{2}{P_{m}} \frac{\Delta \tau}{(\Delta Y)^{2}} \leq 1 \text { and } S_{u} \frac{\Delta \tau}{\Delta Y}+\frac{2}{P_{r}} \frac{\Delta \tau}{(\Delta Y)^{2}}-\Delta \tau \beta \theta^{p-1} \leq 1
\end{gathered}
$$

\section{Results and Discussion}

In this paper, the effect of Hall current on ECF flow along a rotating infinite vertical porous plate in the presence of transverse magnetic field has been investigated using the explicit finite difference iteration technique. To study the physical situation of the developed problem, it has been obtained for the numerical values of the primary velocity $U$, secondary velocity $W$, angular velocity $N$, primary induced magnetic field $H_{1}$, secondary induced magnetic field $H_{3}$ and temperature $\theta$ within the boundary layer for the laminar boundary layer flow. For brevity, only the effects of Hall parameter $\beta_{e}$, Magnetic diffusivity number $P_{m}$, microrotation number $\Delta$ and spin gradient viscosity $\Lambda$ on velocities, induced magnetic fields, temperature as well as shear stresses, couple stress, current densities and Nusselt number are illustrated.

\subsection{Mesh Sensitivity Analysis}

To verify the effects of grid space for $n$, the computations have been carried out for three different grid spaces such as $n=575, n=675$ and $n=775$ are shown in Figure 3. But a negligible change has been seen among these primary velocity profiles, which proves the accuracy of the taken mesh. 


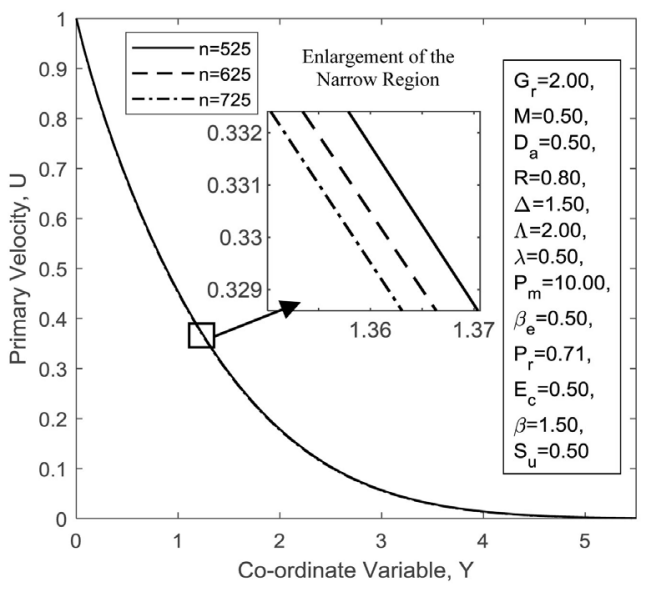

Figure 3. Illustration of primary velocity for different values of Grid Space $n$.

\subsection{Code Validation Test}

The verification of the finite difference solutions by Studio Developer FORTRAN (SDF) 6.6a is achieved with the finite difference solutions by MATLAB R2018a. The same results are obtained from the above-mentioned tools. The computations for Primary velocity $U$ have been shown in Figure 4.

\subsection{Steady-State Solution}

In order to verify the effects of time step size $\Delta \tau$, the computations have been carried out for four different time step sizes as $\tau=1, \tau=20, \tau=30$ and $\tau=40$. It is observed that the result of computations for $U, W, N, H_{1}, H_{3}$ and $\theta$, however, shows so few changes after $\tau=20$ also shows a negligible change after $\tau=30$. Thus the solutions of all variables for $\tau=30$ are essentially the steady-state solutions, which are shown in Figure 5 for primary velocity curves.

\subsection{Effect of Parameters}

In order to get the clear concept of physical properties of the problem, the effects of four parameters namely Hall parameter $\beta_{e}$, Magnetic diffusivity number $P_{m}$, spin gradient viscosity $\Lambda$ and vortex viscosity $\lambda$ of primary velocity $(U)$, shear stress along $x$ direction $\left(\tau_{x}\right)$, secondary velocity $(W)$, shear stress along $z$ direction $\left(\tau_{z}\right)$, angular velocity $(N)$, couple stress, primary induced magnetic field $\left(H_{1}\right)$, current density along $x$ direction $\left(J_{x}\right)$, secondary induced magnetic field $\left(H_{3}\right)$, current density along $z$ direction $\left(J_{z}\right)$, temperature $(\theta)$ and Nusselt number $\left(N_{u}\right)$ have been computed within the boundary which represent graphically through Figures 6-17. For brevity, the effects of other parameters are not shown. For better visualization, some enlargements have been shown for different narrow regions.

Figure 6(a) displays the effect of $\beta_{e}$ on the primary velocity and Figure 7(a) displays the effect of $\beta_{e}$ on the shear stress along $x$ direction for different $P_{m}$. Primary velocity and shear stress along $x$ direction increase for the increasing values of $\beta_{e}$ and $P_{m}$. Figure 6(b) displays the effect of $\Lambda$ on the primary velocity 

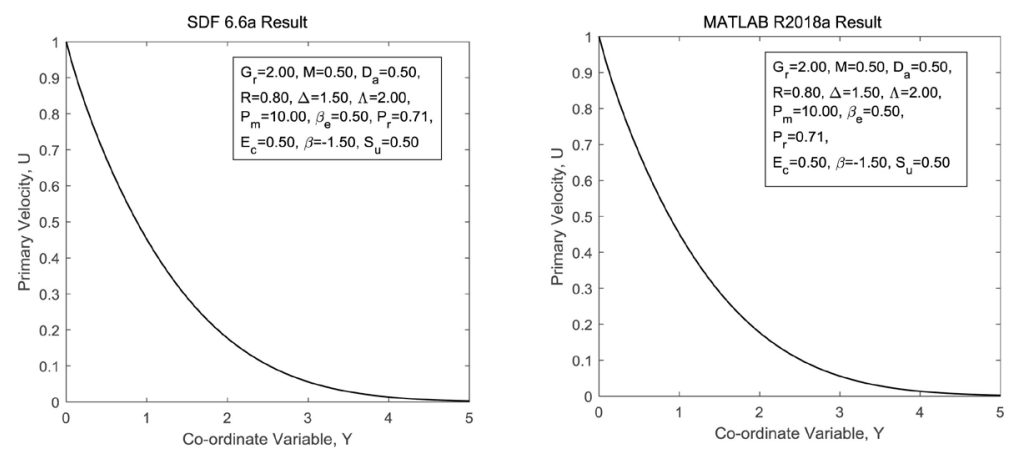

Figure 4. Comparison between SDF 6.6a Results with MATLAB 2018a Results of Primary Velocity $U$.

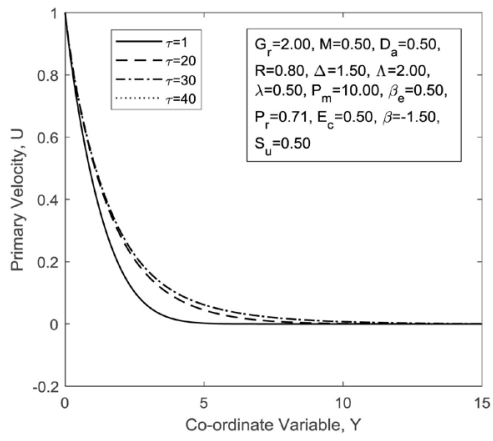

Figure 5. Illustration of primary velocity for different values of dimensionless time $\tau$.

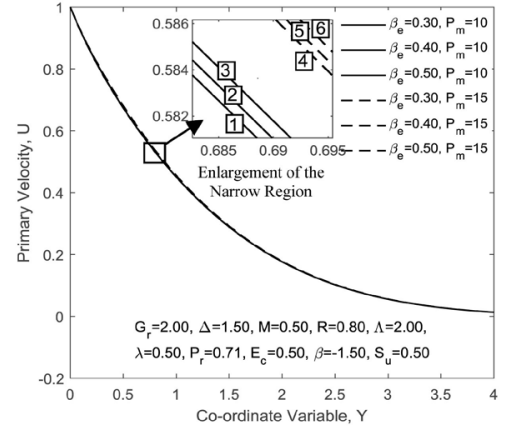

(a)

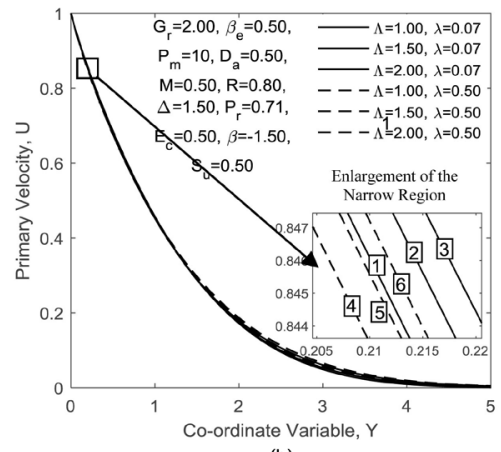

(b)

Figure 6. Primary velocity for various values of (a) $\beta_{e}$ and $P_{m}$ (b) $\Lambda$ and $\lambda$.

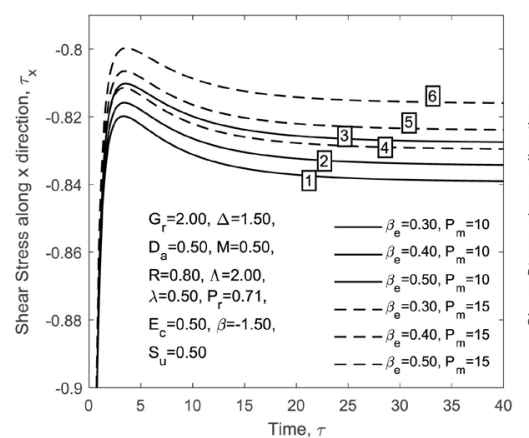

(a)

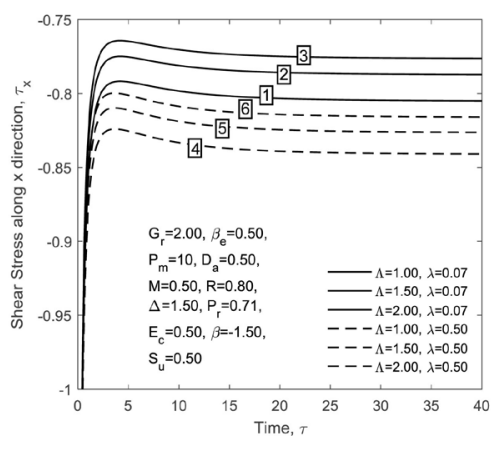

(b)

Figure 7. Shear stress along $x$ direction for various values of (a) $\beta_{e}$ and $P_{m}$ (b) $\Lambda$ and $\lambda$. 


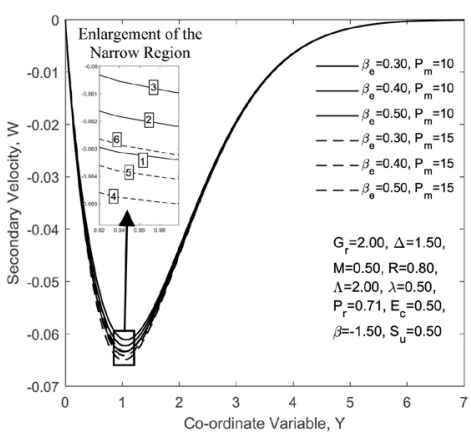

(a)

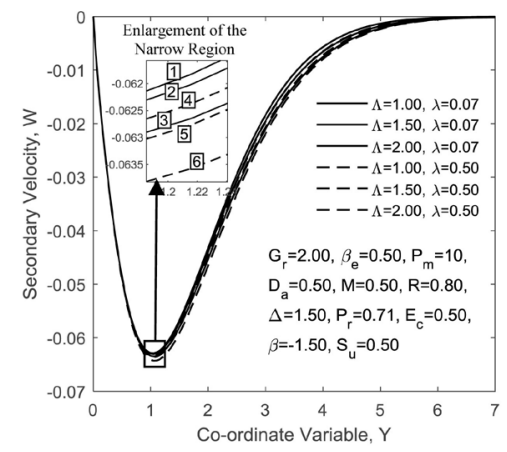

(b)

Figure 8. Secondary velocity for various values of (a) $\beta_{e}$ and $P_{m}$ (b) $\Lambda$ and $\lambda$.

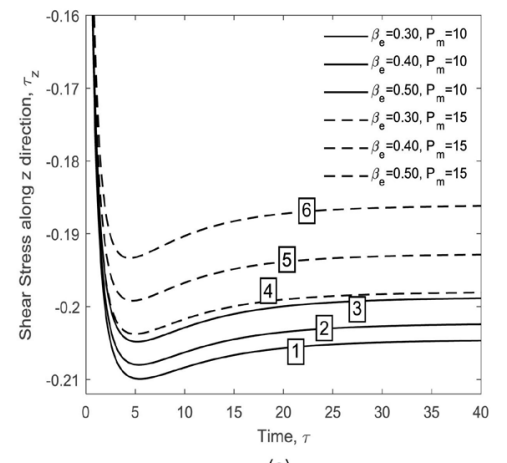

(a)

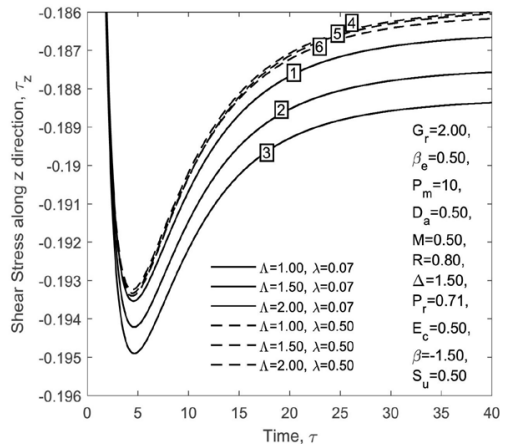

(b)

Figure 9. Shear stress along $z$ direction for various values of (a) $\beta_{e}$ and $P_{m}$ (b) $\Lambda$ and $\lambda$.

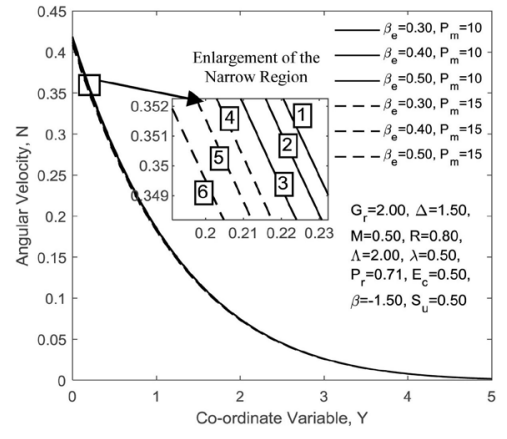

(a)

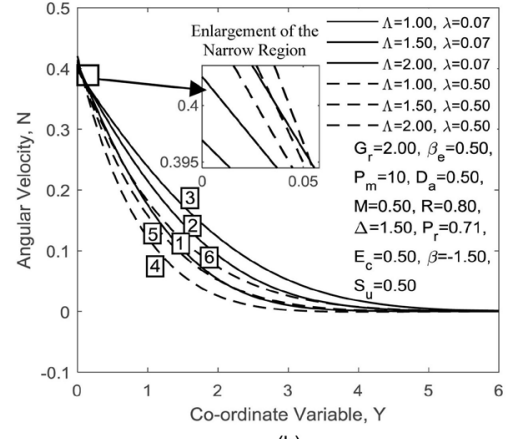

(b)

Figure 10. Angular velocity for various values of (a) $\beta_{e}$ and $P_{m}$ (b) $\Lambda$ and $\lambda$.

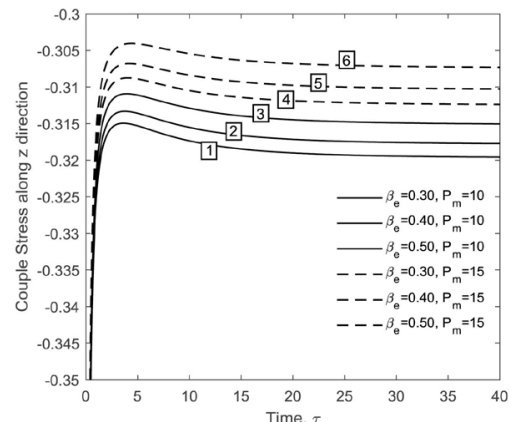

(a)

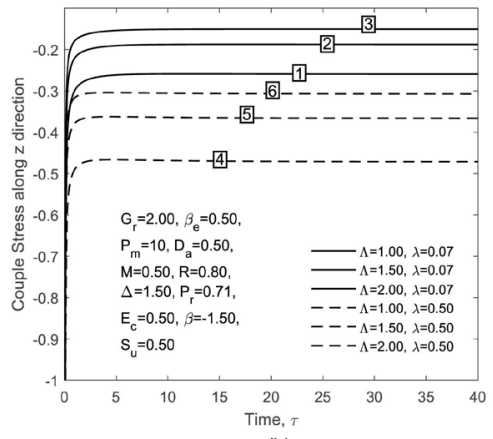

(b)

Figure 11. Couple stress for various values of (a) $\beta_{e}$ and $P_{m}$ (b) $\Lambda$ and $\lambda$. 


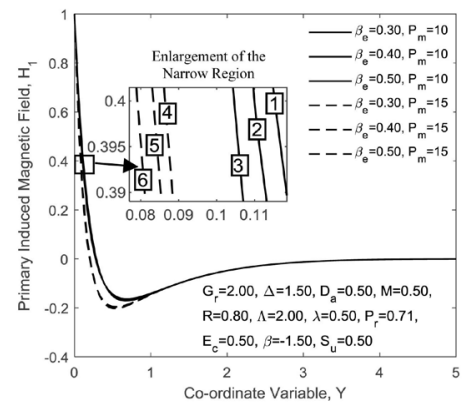

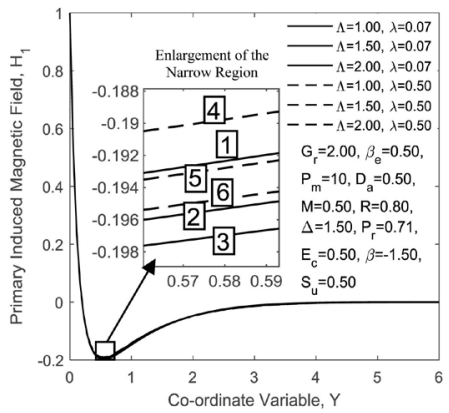

(b)

Figure 12. Primary induced magnetic field for various values of (a) $\beta_{e}$ and $P_{m}$ (b) $\Lambda$ and $\lambda$.
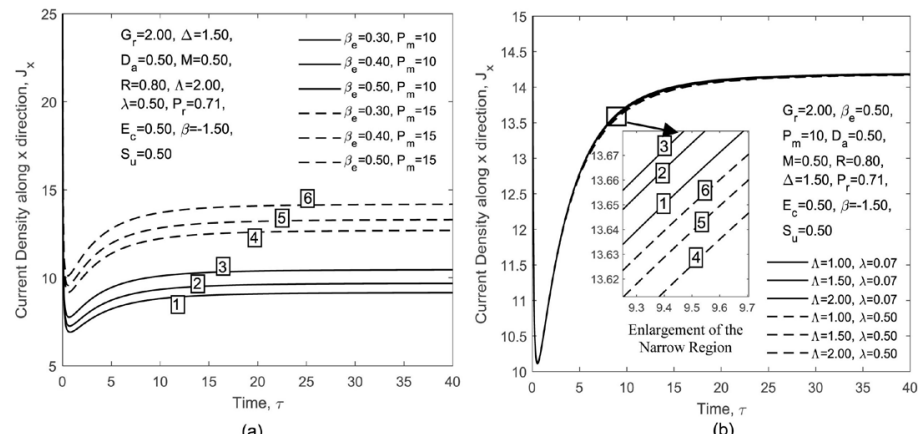

Figure 13. Current density along $x$ direction for various values of (a) $\beta_{e}$ and $P_{m}$ (b) $\Lambda$ and $\lambda$.

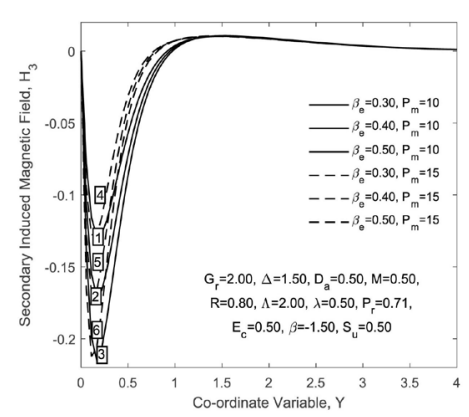

(a)

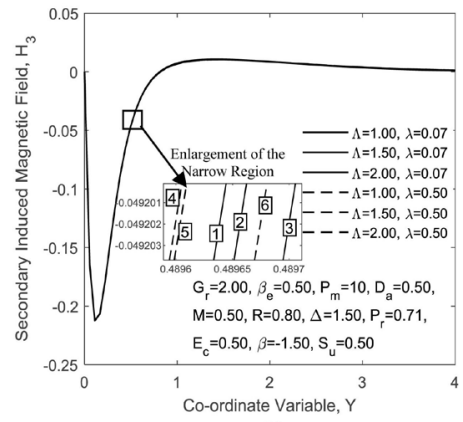

Figure 14. Secondary induced magnetic field for various values of (a) $\beta_{e}$ and $P_{m}$ (b) $\Lambda$ and $\lambda$.

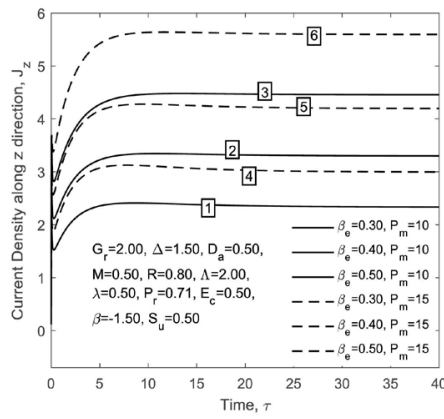

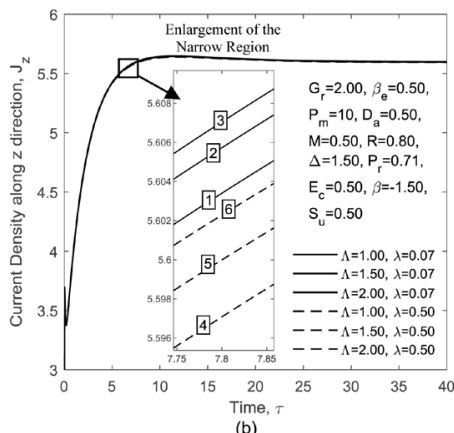

(b)

Figure 15. Current density along $z$ direction for various values of (a) $\beta_{e}$ and $P_{m}$ (b) $\Lambda$ and $\lambda$. 


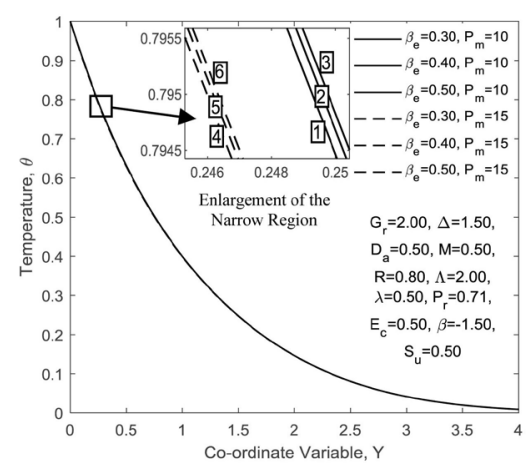

(a)

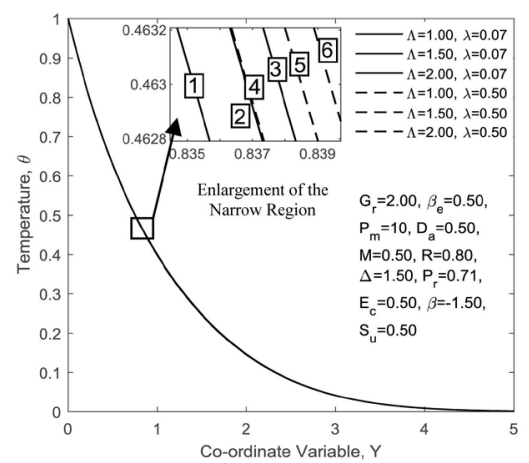

(b)

Figure 16. Temperature for various values of (a) $\beta_{e}$ and $P_{m}$ (b) $\Lambda$ and $\lambda$.

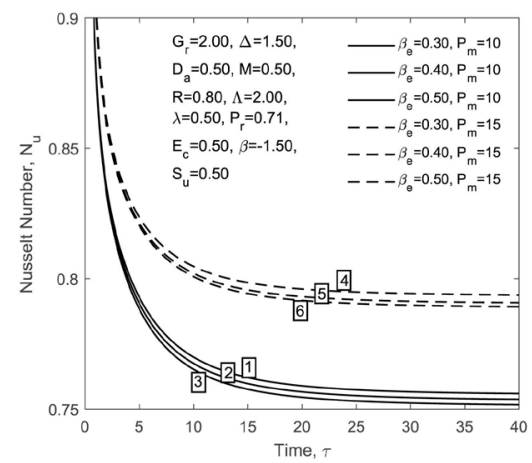

(a)

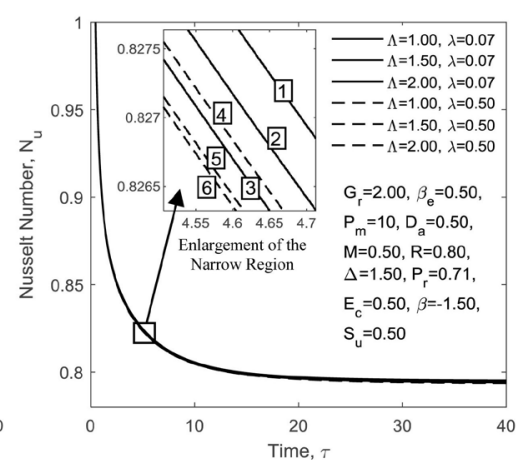

(b)

Figure 17. Nusselt Number for various values of (a) $\beta_{e}$ and $P_{m}$ (b) $\Lambda$ and $\lambda$.

and Figure 7(b) displays the effect of $\Lambda$ on the shear stress along $x$ direction for different $\lambda$. Primary velocity and shear stress along $x$ direction decreases for the increasing values of $\lambda$ and increases for the increasing values of $\Lambda$, where the enlargement of the narrow region denotes the clear visualization.

Figure 8(a) displays the effect of $\beta_{e}$ on the secondary velocity and Figure 9(a) displays the effect of $\beta_{e}$ on the shear stress along $z$ direction for different $P_{m}$. Secondary velocity and shear stress along $z$ direction increase for the increasing values of $\beta_{e}$ and $P_{m}$. Figure 8(b) displays the effect of $\Lambda$ on the secondary velocity and Figure 9(b) displays the effect of $\Lambda$ on the shear stress along $z$ direction for different $\lambda$. Secondary velocity and shear stress along $z$ direction decrease for the increasing values of $\Lambda$ and increases for the increasing values of $\lambda$.

Figure 10(a) displays the effect of $\beta_{e}$ on the angular velocity for different $P_{m}$ Angular velocity decreases for the increasing values of $\beta_{e}$ and $P_{m}$. Figure 10(b) displays the effect of $\Lambda$ on the angular velocity for different $\lambda$. Angular velocity increases for the increasing values of $\Lambda$ and decreases for the increasing values of $\lambda$. Figure 11(a) displays the effect of $\beta_{e}$ on the couple stress for different $P_{m}$. Couple stress increases for the increasing values of $\beta_{e}$ and $P_{m}$. Figure 11(b) displays the effect of $\Lambda$ on the couple stress for different $\lambda$. Couple Stress decreases for the increasing values of $\lambda$ and increases for the increasing values of $\Lambda$.

Figure 12(a) displays the effect of $\beta_{e}$ on the primary induced magnetic field 
for different $P_{m}$. Primary induced magnetic field decreases for the increasing values of $\beta_{e}$ and $P_{m}$. Figure 12(b) displays the effect of $\Lambda$ on the primary induced magnetic field for different $\lambda$. Primary induced magnetic field decreases for the increasing values of $\Lambda$ and increases for the increasing values of $\lambda$. Figure 13(a) displays the effect of $\beta_{e}$ on the current density along $x$ direction for different $P_{m}$. Current density along $x$ direction increases for the increasing values of $\beta_{e}$ and $P_{m}$. Figure 13(b) displays the effect of $\Lambda$ on the current density along $x$ direction for different $\lambda$. Current density along $x$ direction increases for the increasing values of $\Lambda$ and decreases for the increasing values of $\lambda$.

Figure 14(a) displays the effect of $\beta_{e}$ on the secondary induced magnetic field for different $P_{m}$. Secondary induced magnetic field decreases for the increasing values of $\beta_{e}$ and increases for the increasing values of $P_{m}$. Figure 14(b) displays the effect of $\Lambda$ on the secondary induced magnetic field for different $\lambda$. Secondary induced magnetic field decreases for the increasing values of $\Lambda$ and increases for the increasing values of $\lambda$. Figure 15(a) displays the effect of $\beta_{e}$ on the current density along $z$ direction for different $P_{m}$. Current density along $z$ direction increases for the increasing values of $\beta_{e}$ and $P_{m}$. Figure 15(b) displays the effect of $\Lambda$ on the current density along $z$ direction for different $\lambda$. Current density along $z$ direction increases for the increasing values of $\Lambda$ and decreases for the increasing values of $\lambda$.

Figure 16(a) displays the effect of $\beta_{e}$ on the temperature for different $P_{m}$. Temperature increases for the increasing values of $\beta_{e}$ and decreases for the increasing values of $P_{m}$. Figure 16(b) displays the effect of $\Lambda$ on the temperature for different $\lambda$. Temperature increases for the increasing values of $\Lambda$ and $\lambda$.

Figure 17(a) displays the effect of $\beta_{e}$ on the Nusselt number for different $P_{m}$. Nusselt number decreases for the increasing values of $\beta_{e}$ and increases for the increasing values of $P_{m}$. Figure 17(b) displays the effect of $\Lambda$ on the Nusselt number for different $\lambda$. Nusselt number decreases for the increasing values of $\Lambda$ and $\lambda$.

\section{Conclusions}

This paper has investigated the effect of transverse magnetic field and Hall current on the boundary layer heat transfer flow along a vertical infinite porous plate. Numerical calculations are carried out for various values of the dimensionless parameters. Some important findings of this study are given below:

1) The shear stress in $x$ direction increases with the increasing of $\beta_{e}, P_{m}, \Lambda$ and decreases with the increasing of $\lambda$.

2) The shear stress in $z$ direction increases with the increasing of $\beta_{e}, P_{m}, \lambda$ and decreases with the increasing of $\Lambda$.

3) The couple stress increases with the increasing of $\beta_{e}, P_{m}, \Lambda$ and decreases with the increasing of $\lambda$.

4) The current density in $x$ direction increases with the increasing of $\beta_{e}, P_{m}, \Lambda$ and decreases with the increasing of $\lambda$. 
5) The current density in $z$ direction increases with the increasing of $\beta_{e}, P_{m}, \Lambda$ and decreases with the increasing of $\lambda$.

6) The Nusselt number increases with the increasing of $P_{m}$ and decreases with the increasing of $\beta_{e}, \Lambda$ and $\lambda$.

\section{Funding}

This work is financed and supported by National Science and Technology under Ministry of Science and Technology, Government of the People's Republic of Bangladesh.

\section{Conflicts of Interest}

The authors declare no conflicts of interest regarding the publication of this paper.

\section{References}

[1] Hodgkin, A.L. and Horowicz, P. (1959) The Influence of Potassium and Chloride Ions on the Membrane Potential of Single Muscle Fibers. The Journal of Physiology, 148, 127-160. https://doi.org/10.1113/jphysiol.1959.sp006278

[2] Ezzat, M.A. (2004) Free Convection Effects on Extracellular Fluid in the Presence of a Transverse Magnetic Field. Applied Mathematics and Computation, 151, 455-482. https://doi.org/10.1016/S0096-3003(03)00354-0

[3] Eringen, A.C. (1967) Theory of Micropolar Fluids. Indiana University Mathematics Journal, 6, 1-18. https://doi.org/10.1512/iumj.1967.16.16001

[4] Kline, K.A. and Alien, S.J. (1969) Concentration Effects in Oscillatory Blood Flow. Biorheology, 6, 99-108. https://doi.org/10.3233/BIR-1969-6204

[5] Ariman, T. (1971) On the Analysis of Blood Flow. Journal of Biomechanics, 4, 185-192. https://doi.org/10.1016/0021-9290(71)90003-0

[6] Ariman, T., Turk, M.A. and Sylvester, N.D. (1973) Microcontinuum Fluid Mechanics-A Review. International Journal of Engineering Science, 11, 905-930. https://doi.org/10.1016/0020-7225(73)90038-4

[7] Eringen, A.C. (1972) Theory of Thermomicrofluids. Journal of Mathematical Analysis and Applications, 38, 480-496. https://doi.org/10.1016/0022-247X(72)90106-0

[8] Ram, P.C. (1988) Unsteady MHD Free-Convective Flow through a Porous Medium with Hall Currents. Astrophysics and Space Science, 149, 171-174. https://doi.org/10.1007/BF00640477

[9] Garg, B.P. (2012) Combined Effects of Thermal Radiations and Hall Current on Moving Vertical Porous Plate in a Rotating System with Variable Temperature. International Journal of Pure and Applied Mathematics, 81, 335-345.

[10] Kafoussias, N.G. and Williams, E.W. (1995) Thermal-Diffusion and DiffusionThermo Effects on Mixed Free-Forced Convective and Mass Transfer Boundary Layer Flow with Temperature Dependent Viscosity. International Journal of Engineering Science, 33, 1369-1384. https://doi.org/10.1016/0020-7225(94)00132-4

[11] Postelnicu, A. (2004) Influence of a Magnetic Field on Heat and Mass Transfer by Natural Convection from Vertical Surfaces in Porous Media Considering Soret and Dufour Effects. International Journal of Heat and Mass Transfer, 47, 1467-1472. https://doi.org/10.1016/j.ijheatmasstransfer.2003.09.017 
[12] Mohammadein, A.A. and Gorla, R.S.R. (1996) Effects of Transverse Magnetic Field on Mixed Convection in a Micropolar Fluid on a Horizontal Plate with Vectored Mass Transfer. Acta Mechanica, 118, 1-12. https://doi.org/10.1007/BF01410503

[13] Peddiesen, J. and McNItt, R.P. (1970) Boundary Layer Theory for a Micropolar Fluid. Recent Advanced Engineering and Science, 5, 405-426.

https://doi.org/10.1155/2010/627475

[14] Shateyi, S., Mosta, S.S. and Sibanda, P. (2010) The Effects of Thermal Radiation, Hall Currents, Soret, and Dufour on MHD Flow by Mixed Convection over a Vertical Surface in Porous Media. Mathematical Problems in Engineering, 2010, Article ID: 627475. https://doi.org/10.1155/2010/627475

[15] Takhar, H.S., Chamkha, A.J. and Nath, G. (2002) MHD Flow over a Moving Plate in a Rotating Fluid with Magnetic Field, Hall Currents and Free Stream Velocity. International Journal of Engineering Science, 40, 1511-1527. https://doi.org/10.1016/S0020-7225(02)00016-2

[16] Ezzat, M. (2001) Free Convection Effects on Perfectly Conducting Fluid. International Journal of Engineering Science, 39, 799-819.

https://doi.org/10.1016/S0020-7225(00)00059-8

[17] Haque, M.M., Alam, M.M., Ferdows, M. and Al-Mdallal, Q.M. (2013) Numerical Simulation and Stability Analysis on MHD Free Convective Heat and Mass Transfer Unsteady Flow through a Porous Medium in a Rotating System with Induced Magnetic Field. International Journal of Applied Electromagnetic and Mechanics, 41, 121-141. https://doi.org/10.3233/JAE-2012-1598

[18] Ahmed, T. and Alam, M.M. (2013) Finite Difference Solution of MHD Mixed Convection Flow with Heat Generation and Chemical Reaction. Procedia Engineering, 56, 149-156. https://doi.org/10.1016/j.proeng.2013.03.101 